Quim. Nova, Vol. 35, No. 9, 1728-1732, 2012

\title{
ÁCIDOS FENÓLICOS, FLAVONOIDES E ATIVIDADE ANTIOXIDANTE EM MÉIS DE Melipona fasciculata, M. flavolineata (Apidae, Meliponini) E Apis mellifera (Apidae, Apini) DA AMAZÔNIA
}

\author{
Patricia Sertão Oliveira, Regina Celi Sarkis Müller*, Kelly das Graças Fernandes Dantas e Claudio Nahum Alves \\ Faculdade de Química, Instituto de Ciências Exatas e Naturais, Universidade Federal do Pará, Rua Augusto Corrêa, 01, \\ 66075-110 Belém - PA, Brasil
}

Marcus Arthur Marçal de Vasconcelos e Giorgio Cristino Venturieri

Embrapa Amazônia Oriental, Trav. Enéias Pinheiro, s/n, 66017-970 Belém - PA, Brasil

Recebido em 21/11/11; aceito em 6/5/12; publicado na web em 3/8/12

\begin{abstract}
PHENOLIC ACIDS, FLAVONOIDS AND ANTIOXIDANT ACTIVITY IN HONEY OF Melipona fasciculata, M. flavolineata (Apidae, Meliponini) AND Apis mellifera (Apidae, Apini) FROM THE AMAZON. Honey produced by three stingless bee species (Melipona flavolineata, M. fasciculata and Apis mellifera) from different regions of the Amazon was analyzed by separating phenolic acids and flavonoids using the HPLC technique. Data were subjected to multivariate statistical analysis (PCA, HCA and DA). Results showed the three species of honey samples could be distinguished by phenolic composition. Antioxidant activity of the honeys was determined by studying the capacity of inhibiting radicals using DPPH assay. Honeys with higher phenolic compound contents had greater antioxidant capacity and darker color.
\end{abstract}

Keyword: phenolic compounds; honey; HPLC- UV/Vis.

\section{INTRODUÇÃO}

Hoje, na criação de abelhas, existem duas grandes linhas de estudo: a Apicultura e a Meliponicultura. Dentro da Apicultura, o conhecimento sobre o mel já vem sendo construído em várias regiões do Brasil, no entanto, na Meliponicultura esses estudos são mais recentes, sendo desenvolvidos com as abelhas regionais.

O mel de abelha comercializado no mundo todo é em sua quase totalidade oriundo de uma única espécie, a Apis melífera. ${ }^{1}$ Contudo, nas regiões tropicais e subtropicais existem mais de 400 espécies descritas de abelhas sociais que podem produzir mel. Estas outras abelhas são conhecidas como abelhas indígenas sem ferrão, ou meliponíneos. São abelhas dóceis, de fácil manejo e necessitam de pouco investimento para a sua criação. ${ }^{2}$ Dentre todos os gêneros dos meliponíneos, o Melipona é o mais diverso e é na Amazônia que encontramos a maior diversidade. ${ }^{3}$ Além de ser mais diverso, o gênero Melipona é o que abriga as espécies mais produtivas e criadas. ${ }^{4}$

Embora produzindo mel em menor quantidade por colônia, algumas espécies possuem uma população correspondente a $5 \%$ de uma colônia de $A$. melífera. ${ }^{5}$ Os meliponíneos têm o importante papel de fornecer um produto que se diferencia do mel de A. mellifera, principalmente no sabor e aroma peculiares, podendo alcançar preços elevados no mercado. ${ }^{6}$

De maneira geral, o mel das espécies de meliponíneos tem como principal característica a diferenciação nos teores da sua composição, destacando-se o teor de água (umidade), que o torna menos denso que o mel das abelhas africanizadas. ${ }^{7}$ A cor varia do quase transparente ao âmbar escuro e o gosto e os níveis de açúcar dependem da espécie, época, região e, principalmente, da florada. ${ }^{8}$ Além dos açúcares em solução, o mel também contém ácidos orgânicos, enzimas, vitaminas, flavonoides, minerais e uma extensa variedade de compostos orgânicos, que contribuem para sua cor, odor e sabor. ${ }^{9}$

A utilização do mel na nutrição humana não deveria se limitar apenas à sua característica adoçante mas, principalmente, por ser um alimento de alta qualidade, rico em energia e inúmeras outras

*e-mail: reginasarkis@ @otmail.com substâncias benéficas ao equilíbrio dos processos biológicos do nosso corpo. Além de sua qualidade como alimento, esse produto único é dotado de inúmeras propriedades terapêuticas, sendo utilizado pela medicina popular sob diversas formas e associações como fitoterápicos. ${ }^{10}$ Pesquisas relacionadas à identificação de compostos fenólicos em mel, tendo em vista a atividade antioxidante e possível origem botânica, aumentaram consideravelmente após a aplicação de metodologias usando HPLC..$^{11-13}$

Muitos compostos fenólicos têm propriedades captadoras de radicais livres, o que confere a atividade antioxidante. Os radicais livres têm sido considerados como agentes causadores de isquemias cerebral e cardíaca, doenças de Parkinson, distúrbios gastrointestinais, envelhecimento, entre outros. As células vivas possuem capacidade limitada para anular a atividade destes radicais livres, mas se acredita que a ingestão de antioxidantes pode melhorar a proteção das células e, portanto, a sua função fisiológica. Estes antioxidantes são geralmente obtidos a partir da alimentação e incluem vitaminas $C$ e E, $\beta$-caroteno e uma variedade de compostos fenólicos, incluindo ácidos fenólicos e flavonoides. ${ }^{14}$ Estes compostos fenólicos também apresentam atividade antiviral, antibacteriana e anti-inflamatória. ${ }^{15-17}$

Este trabalho objetivou identificar o perfil cromatográfico de ácidos fenólicos e flavonoides utilizando a extração em fase sólida (SPE) e cromatografia líquida de fase reversa (RP-HPLC) e realizar estudo da atividade antioxidante nos méis de três diferentes espécies de abelhas: A. mellifera (africanizada) espécie exótica, Melipona flavolineata (uruçu-amarela) e M. fasciculata (uruçu-cinzenta) espécies nativas. As amostras de mel foram obtidas de quatro municípios do Estado do Pará.

\section{PARTE EXPERIMENTAL}

\section{Reagentes e soluções analíticas}

Como padrões, com grau de pureza superior a 99\%, foram utilizados os ácidos gálico, cafeico, ferulico, $o$-cumárico, $m$-cumárico, p-cumárico, $o$-metoxicinâmico, trans-cinâmico, vanílico e os flavonoides quercetina e miricetina (Sigma, St. Louis, USA), dos quais 
foram preparadas soluções metanólicas (Metanol, Merck, Darmstadt, Germany), com as concentrações de $4,0 \mathrm{mg} \mathrm{mL}^{-1}$. Todas as soluções foram armazenadas a $6{ }^{\circ} \mathrm{C}\left( \pm 1^{\circ} \mathrm{C}\right)$.

As fases móveis foram preparadas pela mistura de metanol e acetonitrila e água ultrapura acidificada (Milli-Q, Millipore ${ }^{\circledR}$ ).

\section{Extração em SPE}

A extração dos ácidos fenólicos foi realizada de acordo com o método descrito por Michalkiewiczs e colaboradores. ${ }^{18}$ Aproximadamente $20 \mathrm{~g}$ de mel foram diluídas em $50 \mathrm{~mL}$ de água ultrapura acidificada ( $\mathrm{pH}$ ajustado para 2,0) com $\mathrm{HCl} 12 \mathrm{~mol} \mathrm{~L}^{-1} \mathrm{e}$ filtrada em algodão a fim de eliminar partículas em suspensão. O filtrado foi adicionado ao cartucho de SPE (Stracta-X, Phenomenex, Torrance, CA, USA) para a separação da fração fenólica que foi adsorvida com $50 \mathrm{~mL}$ de metanol. Após a obtenção do extrato metanólico, este foi concentrado em rota-vapor até volume de $5 \mathrm{~mL}$ a $40{ }^{\circ} \mathrm{C}$. O concentrado foi filtrado em membrana $0,45 \mu \mathrm{m}$ (Millipore, Bedford, MA, USA) e condicionado em frascos de polietileno $(2 \mathrm{~mL})$ à temperatura de $5{ }^{\circ} \mathrm{C}\left( \pm 1^{\circ} \mathrm{C}\right)$ até a análise.

\section{Condições cromatográficas}

A separação cromatográfica foi realizada usando cromatógrafo líquido de alta eficiência modelo Pro Star (Varian, Mulgrave, Austrália), acoplado a um detector UV-Vis. (Varian, Mulgrave, Austrália) trabalhando no comprimento de onda de $290 \mathrm{~nm}$. Para as análises foi utilizada coluna RP-C-18 (Kromasil 100-5 $250 \mathrm{x}$ $4,6 \mathrm{~mm}$ ) e sistema manual de injeção com volume fixo de $20 \mu \mathrm{L}$. A massa para os padrões utilizados foi de $2 \mathrm{mg}$. As fases móveis usadas foram água: ácido acético (99:1, fase A) e metanol:acetonitrila:ácido acético (59:40:1, fase B). Os padrões cromatográficos foram avaliados individualmente e também em misturas. O tempo de eluição, o fluxo da fase móvel e o gradiente de eluição foram otimizados. A melhor separação cromatográfica dos padrões foi obtida usando um fluxo de $0,5 \mathrm{~mL} \mathrm{~min}^{-1}$ e um gradiente de eluição de $0 \mathrm{~min}-22 \% \mathrm{~B}$; 15 min - 30\% B; 17 min - 40\% B; 30 min - 60\% B; 38 min - 30\% B; 50 min $-22 \%$ B e, eluição isocrática com $22 \%$ B até 60 min. Os cromatogramas dos padrões e amostras obtidos por UV foram registrados usando o software do equipamento. O monitoramento dos perfis cromatográficos foi realizado a $290 \mathrm{~nm}$, visto que a maioria dos ácidos fenólicos e flavonoides encontrados nos méis mostra suas absorções máximas no UV, próximo a esse comprimento de onda. ${ }^{19}$ Todas as amostras foram analisadas em triplicata.

\section{Identificação dos ácidos fenólicos e flavonoides}

A identificação dos ácidos fenólicos e flavonoides nos cromatogramas dos méis foi feita por comparação direta dos tempos de retenção, coinjeção e análises das curvas de absorção no UV, obtidas em espectrofotômetro Varian, com as curvas de absorção dos padrões.

\section{Amostras de mel}

Foram analisadas 36 amostras de mel, sendo 12 de cada espécie de $M$. flavolineata (uruçu-amarela), $M$. fasciculata (uruçu-cinzenta) e A. mellifera (africanizada), colhidas no período de baixo índice pluviométrico que ocorre de setembro a fevereiro, possibilitando a exploração dos produtos produzidos pelas abelhas nativas da região. As amostras foram provenientes de quatro municípios da mesorregião do nordeste paraense, que apresenta grande diversidade de solo e espécies botânicas. Os municípios envolvidos neste estudo foram os do nordeste paraense, localizados nas microrregiões Bragantina
(Tracuateua), do Salgado (São João de Pirabas e Vigia) e Guamá (São Miguel do Guamá). As amostras foram coletadas de colméias racionais, conforme modelo recomendado por Venturieri. ${ }^{20}$ Este sistema de criação em caixas racionais facilitou a coleta do mel, tornando-a mais rápida, higiênica e causando poucos distúrbios à colônia. ${ }^{21}$ As análises em cada amostra de mel foram realizadas em triplicata.

\section{Métodos de análise multivariada}

Empregou-se, inicialmente, o método de análise de discriminante, cujo principal objetivo é determinar funções discriminantes usando as medidas variáveis que separam os grupos. A análise discriminante (DA) é um procedimento de classificação que maximiza a variância entre as categorias e miniminiza a variância dentro das categorias. Este método torna um número de funções discriminantes ortogonais igual ao número de categorias menos um. ${ }^{22}$ Em seguida, utilizou-se o método de análise de cluster ou agrupamento hierárquico (HCA), com o intuito de reunir os parâmetros e observações semelhantes e separar os mais distantes. Este método unifica os parâmetros em classes ou grupos, cuja variação das observações é semelhante. Como medida de similaridade foi utilizada a distância euclidiana média e como método hierárquico aglomerativo, o método de Ligação Completa. Com base nos cálculos, foi estabelecido o dendrograma, o qual permite verificar o grau de similaridade entre os genótipos e grupos similares, ou entre dois grupos distintos. Por fim, foi realizada a análise de componentes principais (PCA), ${ }^{23}$ que é uma técnica de análise multivariada cujo objetivo é reduzir a dimensão de problemas multivariados e detectar os parâmetros mais significativos que descrevam o conjunto de dados com a mínima perda dos dados originais. As análises de agrupamento, discriminante e por componentes principais foram realizadas utilizando-se o conjunto de dados autoescalonado com o programa MINITAB R $14 .{ }^{24}$

\section{Cor}

A classificação de cor dos méis foi realizada em espectrofotômetro (Shimadzu modelo UV-160ª a $560 \mathrm{~nm}$ em célula de 1 $\mathrm{cm}$ e usando-se como branco, glicerina pura. Os dados obtidos no espectrofotômetro foram transformados em cor usando-se a Escala de Cores de Pfund. ${ }^{25}$

\section{Atividade antioxidante}

A determinação da atividade antioxidante foi conduzida usando solução metanólica 0,3 mM de DPPH (2,2-difenilpicril-hidrazila). Na presença de um antioxidante, a coloração púrpura do DPPH decai e a mudança de absorbância pode ser lida através da espectrometria. As soluções estoque dos méis $\left(100 \mathrm{mg} \mathrm{mL}^{-1}\right)$ e dos padrões (vitamina $\mathrm{C}$ e Trolox) foram preparadas em metanol, que foram diluídas em diversas concentrações e transferidas para cubetas, juntamente com a solução metanólica de DPPH. Após 30 min de repouso no escuro, a determinação foi realizada usando um espectrofotômetro UV-Vis. no comprimento de onda de $517 \mathrm{~nm}$. Dois ensaios independentes foram realizados e todos em triplicata. A atividade antioxidante foi expressa em valores de concentração efetiva em $50 \%$ do total do efeito $\left(\mathrm{CE}_{50}\right)$, através do gráfico que relaciona o percentual de atividade com a concentração da substância ensaiada.

\section{RESULTADOS E DISCUSSÃO}

As condições cromatográficas foram otimizadas para o sistema com ajuste de fluxo de $0,5 \mathrm{~mL}$ min. ${ }^{-1}$ Para as fases móveis foram testadas concentrações decrescentes de ácido acético e metanol e 
a estas adicionada acetonitrila (1\%) para se obter um aumento da polaridade. Nestas condições foi obtida a melhor separação entre os compostos fenólicos, apresentados na Figura 1. Os compostos fenólicos analisados foram preparados na concentração de $4 \mathrm{mg} \mathrm{mL}^{-1}$, utilizando como solvente o metanol em grau HPLC. No método foi mantido o comprimento de onda de $290 \mathrm{~nm}$, volume de injeção de $20 \mu \mathrm{L}$ e tempo total de corrida de $60 \mathrm{~min}$.

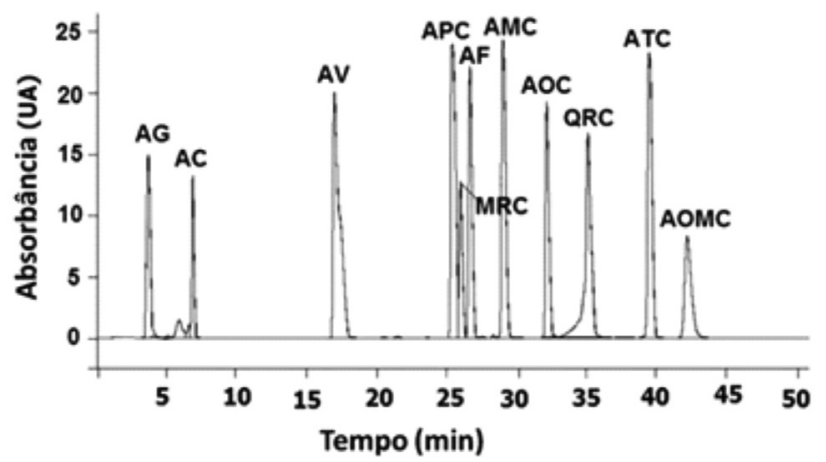

Figura 1. Perfil cromatográfico por CLAE da mistura dos padrões de ácidos fenólicos e flavonoides a $290 \mathrm{~nm}$. Os compostos fenólicos são: (AG) ácido gálico, (AC) ácido cafeico, (AV) ácido vanílico, (APC) ácido p-cumárico, $(M R C)$ mircetina, $(A F)$ ácido ferulico, (AMC) ácido m-cumárico, (AOC) ácido o-cumárico, (QRC) Quercetina, (ATC) ácido trans-cinâmico e (AOMC) ácido o-metoxinâmico

Os tempos de retenção $\left(\mathrm{t}_{\mathrm{R}}\right)$ obtidos para os padrões de ácido gálico (AG), ácido cafeico (AC), ácido vanílico (AV), ácido p-cumárico (APC), miricetina(MRC), ácido ferulico (AF), ácido m-cumárico (AMC), ácido o-cumárico (AOC), quercetina $(\mathrm{QRC})$, ácido trans-cinâmico (ATC), ácido o-metoxicinâmico (AOMC) foram 4,75; 6,$70 ; 16,82 ; 23,26 ; 25,24 ; 26,51 ; 28,86 ; 31,99 ; 34,38 ; 39,31$ e 42,03 min, respectivamente.

\section{Perfis fenólicos em méis de $A$. mellifera}

A análise dos ácidos fenólicos e flavonoides por HPLC nos méis coletados na região de Tracuateua mostrou maior representatividade de ácido gálico (35\% do total da quantidade de ácidos fenólicos). Os ácidos ferrúlico, desconhecido $\left(\mathrm{Ph}_{1}\right), p$-cumárico e quercetina são considerados compostos secundários e encontraram-se presentes na matriz numa proporção de 15,$7 ; 15,5 ; 11,2$ e $11,1 \%$, respectivamente. Os demais ácidos fenólicos presentes nos méis analisados obtiveram porcentagem baixa em relação à proporção total de ácidos fenólicos, com valores de $8 \%$ para ácido vanílico e $0,98 \%$ para ácido $m$-cumárico.

O perfil cromatográfico obtido para os méis da região de São João de Pirabas apresentou como compostos fenólicos principais a quercetina e os ácidos gálico e $o$-cumárico. A quercetina representa $33,4 \%$, enquanto os ácidos $o$-cumárico e gálico representam 29,8 e 24,5\% do total de ácidos fenólicos, respectivamente. O ácido ferrúlico, o ácido fenólico desconhecido $\mathrm{Ph}_{2}$ e o ácido trans-cinâmico estão presentes em baixos níveis. Estes três compostos fenólicos majoritários representam $87,7 \%$ do total de fenólicos presentes no mel.

Os méis da região de São Miguel do Guamá mostraram a partir do cromatograma obtido que o principal ácido fenólico em sua composição foi o ácido gálico, com $69,7 \%$. O ácido fenólico desconhecido $\left(\mathrm{Ph}_{1}\right)$ e o ácido $p$-cumárico apresentaram 10,3 e 4,1\%, respectivamente.

No município de Vigia, os perfis cromatográficos obtidos para os méis coletados mostraram como ácido fenólico principal o ácido gálico, com 79,6\%. A quercetina e os ácidos $p$-cumárico, o-cumárico e trans-cinâmico obtiveram valores que correspondem a 5,7; 5,3; 2,8 e $0,6 \%$, respectivamente.

\section{Perfis fenólicos em méis de $M$. flavolineata}

Os cromatogramas obtidos para os méis coletados no município de Tracuateua mostraram que a quercetina representa $67,8 \%$ do total de compostos fenólicos presente nas amostras, seguido pelos ácidos o-cumárico e gálico como componentes secundários com 16,5 e $13,9 \%$, respectivamente. Os demais, ácido $m$-cumárico e $o$-cumárico, são encontrados em pequenas quantidades.

Em relação aos méis de $A$. mellifera coletados nesta mesma região, foi observada uma diferença na composição do perfil fenólico, onde o ácido gálico foi o composto fenólico dominante. Isto ocorre, devido à possível preferência destas espécies por origens botânicas diferentes.

O perfil de compostos fenólicos de méis da região de São João de Pirabas é dominado pelos compostos fenólicos quercetina, ácidos gálico e $o$-cumárico. Este perfil fenólico foi semelhante ao encontrado em méis da espécie $A$. mellifera da mesma região, nos quais a quercetina representa $31,3 \%$, enquanto os ácidos gálico e $o$-cumárico compõem 23,8 e 23,6\% do total de compostos fenólicos, respectivamente. Os ácidos fenólico desconhecido $\mathrm{Ph}_{2}, p$-cumárico, ferúlico e trans-cinâmico também estão presentes, porém, em baixos teores, representando $21,3 \%$ do total de fenólicos.

No município de Vigia, os méis coletados apresentaram perfis cromatográficos contendo ácido gálico como ácido fenólico principal com $34,2 \%$ e a quercetina com $30,4 \%$. Os ácidos fenólicos desconhecidos $\left(\mathrm{Ph}_{2}\right.$ e $\left.\mathrm{Ph}_{3}\right)$,o-cumárico e $p$-cumárico apresentaram valores inferiores de 13,2; 5,8 e 3,6\%, respectivamente. As espécies A. mellifera e M. flavolineata desta região diferenciam-se em relação à espécie botânica visitada, devido à diferença significativa na quantidade de compostos fenólicos presentes no perfil cromatográfico dos méis analisados.

\section{Perfis fenólicos em méis de $M$. fasciculata}

Os cromatogramas obtidos para os méis coletados na região de Tracuateua mostraram um teor de $80,7 \%$ de ácido gálico. O ácido vanílico e a quercetina obtiveram teores inferiores em relação à proporção total de compostos fenólicos presentes com valores de $12 \%$ para quercetina e 6,3\% para ácido vanílico. O perfil cromatográfico encontrado nas amostras indica a presença de apenas três compostos fenólicos, sendo o ácido gálico predominante. A espécie A. mellifera desta região também apresenta como composto fenólico principal o ácido gálico. Apenas a espécie M. flavolineata possui característica diferenciada, sendo o ácido gálico o componente secundário.

Os perfis fenólicos observados nos méis da região de São João de Pirabas apresentaram como compostos fenólicos principais o ácido gálico e a quercetina. O ácido gálico representa 39,2\%, enquanto a quercetina apresenta teor de $26,7 \%$ do total de compostos fenólicos. Os ácidos $o$-cumárico, $p$-cumárico, fenólico desconhecido $\mathrm{Ph}_{2}$ e ferrúlico estão presentes em baixas quantidades 16,9; 14,8; 12,3 e 1,1\%, respectivamente. As espécies A. mellifera e M. flavolineata desta região apresentam características semelhantes, diferenciando-se apenas no percentual individual dos compostos fenólicos predominantes.

O perfil cromatográfico obtido para os méis da região de São Miguel do Guamá apresentou como composto fenólico identificado apenas o ácido gálico, com $2 \%$ do total de compostos fenólicos. $\mathrm{O}$ composto fenólico principal foi o ácido desconhecido $\left(\mathrm{Ph}_{1}\right)$ com 97 , $8 \%$. Os méis da espécie $A$. mellifera apresentaram maior variedade de compostos, tendo como principal componente o ácido gálico com $69,7 \%$. 


\section{Tratamento estatístico}

\section{Análise discriminante $(A D)$}

A análise discriminante foi realizada para determinar previamente as variáveis (compostos fenólicos) mais significantes na separação das espécies estudadas. Optou-se pelo método de análise discriminante passo a passo (stepwise discriminant analysis), o qual adiciona ou remove variáveis do modelo. Desta forma, obtiveram-se 6 variáveis mais significantes entre as 13 investigadas: $\mathrm{AG}, \mathrm{AOC}, \mathrm{PH}_{3}, \mathrm{QRC}, \mathrm{AV}$ e AC. As funções discriminantes são dadas abaixo:

Grupo A: -14.08 -9,55 AG -27,33 AOC +27,30 $\mathrm{PH}_{3}+54,35$ QRC $+6,04 \mathrm{AV}-7,96 \mathrm{AC}$

Grupo B: $-14.59+9,55 \mathrm{AG}+33,49 \mathrm{AOC}+18,25 \mathrm{PH}_{3}-50,59 \mathrm{QRC}$ $-7,77 \mathrm{AV}+0,32 \mathrm{AC}$

Grupo C: -6,32 -3,51 AG -16,48 AOC -69,15 $\mathrm{PH}_{3}+28,34 \mathrm{QRC}+4,82$ $\mathrm{AV}+3,05 \mathrm{AC}$

onde Grupo A refere-se à espécie A. mellifera, Grupo B à M. flavolineata e Grupo C à M. fasciculata.

A matriz de classificação obtida aplicando-se o método de análise discriminante linear mostrou que as porcentagens de acerto do modelo foram de $100 \%$. Portanto, as variáveis $\mathrm{AG}, \mathrm{AOC}, \mathrm{PH}_{3}, \mathrm{QRC}, \mathrm{AV}$ e $\mathrm{AC}$ são capazes de distinguir as três espécies estudadas, com $100 \%$ de acerto. Estas representam os compostos fenólicos majoritários nas amostras de mel estudadas.

\section{Análise de agrupamento hierárquico (HCA)}

A análise de clusters (AC) foi aplicada para os dados autoescalonados, empregando-se a distância euclidiana para calcular as similaridades das amostras e foi utilizado um procedimento de agrupamento hierárquico para estabelecer os clusters. Os resultados obtidos podem ser observados no dendograma da Figura 2, onde ocorrem cinco agrupamentos distintos (A, B. C, D e E) com similaridade de aproximadamente $41 \%$. Da esquerda para a direita, o agrupamento A formado com similaridade de 42,42\% constitui-se das espécies A. mellifera (AT), M. flavolineata, popularmente conhecida como uruçu-amarela (UA-T) e $M$. fasciculata, também conhecida como uruçu-cinzenta

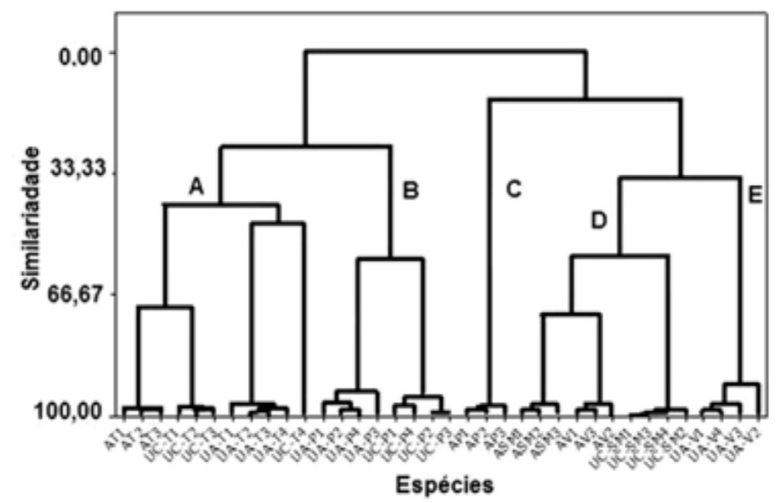

Figura 2. Dendrograma obtido com as seis variáveis selecionadas por AD
(UC-T), sendo as amostras pertencentes à região de Tracuateua (T); o agrupamento B, com similaridade de 56,73\%, contém as amostras de mel das espécies de abelha nativa sem ferrão (UA-P e UC-P), correspondentes à região de São João de Pirabas $(\mathrm{P})$; o agrupamento $\mathrm{C}$, com similaridade de $12,86 \%$, é formado pelas amostras de mel da espécie A. mellifera (AP) da região de São João de Pirabas; o agrupamento D, com similaridade de 56,42\%, corresponde às amostras de A. mellifera (ASM e AV) das regiões de São Miguel do Guamá (SM) e Vigia (V) e M. fasciculata de São Miguel do Guamá; por fim, o agrupamento E, com similaridade de $34,37 \%$, é formado pelas amostras produzidas pela espécie M. flavolineata (UA-V) da região de Vigia.

\section{Análise de componente principal}

A análise de componentes principais obteve resultado semelhante aos encontrados na análise de cluster. Empregando dados autoescalonados observou-se a formação de 5 grupos bem definidos, caracterizando uma diferença entre as espécies de abelhas com e sem ferrão e suas respectivas regiões de coleta. A projeção das duas primeiras componentes é apresentada na Figura 3.

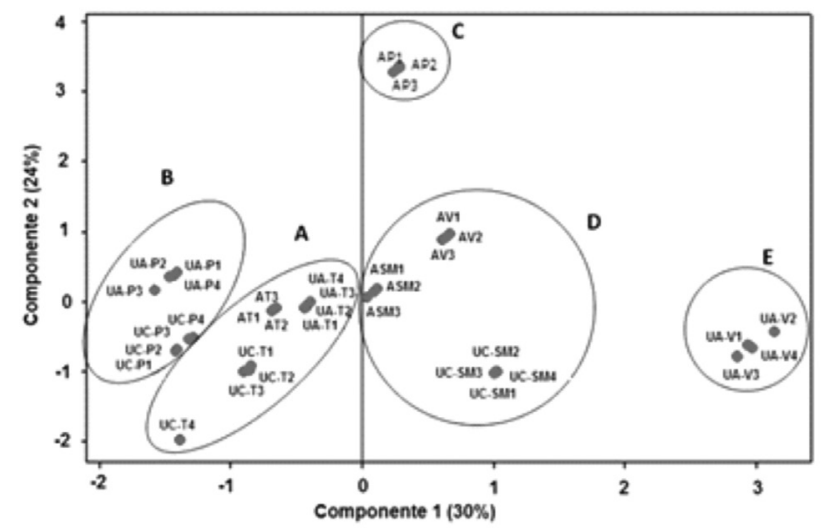

Figura 3. Análise de componentes principais das amostras de mel

As duas primeiras componentes principais conseguem juntas explicar 54\% do total da variância e podem ser consideradas suficientes para descrever os dados.

Baseado nos resultados obtidos das análises de HCA e PCA conclui-se que as porcentagens de ácido gálico (AG), ácido o-cumárico (AOC), ácido desconhecido $\left(\mathrm{PH}_{3}\right)$, quercetina (QRC), ácido vanílico (AV) e ácido cafeico (AC) são capazes de separar as amostras de mel das diferentes espécies de abelha de diferentes regiões do nordeste paraense.

Cor

As amostras analisadas estão dentro dos padrões exigidos pela Legislação Brasileira, que classifica o mel do incolor ao âmbar escuro (Tabela 1).

Tabela 1. Valores de cor, teores totais de fenóis - PT (mg de ácido gálico $100 \mathrm{~g}^{-1}$ de mel) e $\mathrm{CE}_{50}\left(\mathrm{mg} 100 \mathrm{~g}^{-1}\right)$ obtidos para os méis de A de A. mellifera, Melipona flavolineata e M. fasciculata

\begin{tabular}{lccccccccc}
\hline \multirow{2}{*}{ Localidades } & \multicolumn{3}{c}{ A. mellifera } & \multicolumn{3}{c}{ M. flavolineata } & \multicolumn{3}{c}{ M. fasciculata } \\
\cline { 2 - 9 } & Cor* & PT & CE $_{50}$ & Cor* & PT & CE $_{50}$ & Cor* $^{*}$ PT & CE $_{50}$ \\
\hline Tracuateua & A & 68,25 & 29,31 & AC & 26,39 & 48,92 & EAC & 25,53 & 54,43 \\
São João de Pirabas & A & 138,25 & 9,13 & A & 236,71 & 6,85 & A & 88,81 & 15,58 \\
São Miguel do Guamán & A & 154,28 & 8,87 & - & - & - & AC & 59,78 & 31,04 \\
Vigia & AC & 36,68 & 41,76 & EAC & 56,78 & 32,03 & - & - & - \\
\hline
\end{tabular}




\section{Atividade antioxidante}

Os melhores resultados obtidos para atividade antioxidante foram para os méis que apresentaram os teores mais elevados de polifenóis totais e coloração mais escura (Tabela 1).

Os compostos majoritários identificados por cromatografia líquida nestas amostras foram, respectivamente, os ácidos gálico, $O$-cumárico e $p$-cumárico e a quercetina. Os méis da espécie A. mellifera das regiões de São João de Pirabas e São Miguel do Guamá mostraram melhor inibição do radical DPPH, tendo atividade antioxidante significativa em relação às demais espécies, exceto para méis de M. flavolineata da região de São João de Pirabas que obtiveram o melhor percentual de inibição, com média de $6,85 \%$.

Para méis de meliponíneos apenas as amostras coletadas no município de São João de Pirabas apresentaram melhores atividades antioxidantes. Os méis das espécies $M$. flavolineata e $M$. fasciculata das regiões de São Miguel do Guamá e Vigia, apesar dos altos teores de polifenóis totais, tiveram os resultados menos significativos de $\mathrm{CE}_{50}$. Os polifenóis presentes em menor concentração também tiveram menor influência na atividade antioxidante dos méis, bem como a coloração variando do âmbar claro ao extra-âmbar claro. A correlação entre a $\mathrm{CE}_{50}$ e o conteúdo de fenólicos totais foi estatisticamente significativa, sendo obtido um coeficiente de correlação de 0,87 .

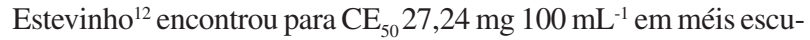

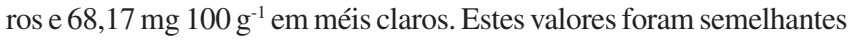
aos encontrados neste estudo, onde os méis mais escuros possuem melhores atividades antioxidantes. Méis analisados no Rio de Janeiro apresentaram concentração efetiva em $50 \%$ do total do efeito $\left(\mathrm{CE}_{50}\right)$ variando de 4,82 a $77,05 \%$. Lianda ${ }^{13}$ obteve para méis de $A$. mellifera de regiões do Rio de Janeiro valores de $\mathrm{CE}_{50}$ variando de 10,81 a

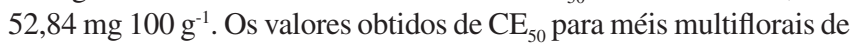
diversas regiões da África do Sul variaram de 1,63 a 29,13 $\mathrm{mg} \mathrm{mL}^{-1} .^{14}$

\section{CONCLUSÃO}

O método HPLC em conjunto com o método SPE de extração foi sensível na separação dos compostos fenólicos presentes nas amostras. Das 36 amostras de mel analisadas foram observados a presença de 13 compostos fenólicos, sendo 3 não identificados $\left(\mathrm{Ph}_{1}, \mathrm{Ph}_{2} \mathrm{e} \mathrm{Ph}_{3}\right)$. Para a maioria dos méis analisados foram identificados como compostos majoritários o ácido gálico e a quercetina.

A aplicação da técnica de AD permitiu a elaboração de um modelo com porcentagem de predição de $100 \%$ na classificação das espécies. Os métodos AC e ACP foram capazes de distinguir as espécies estudadas através da composição fenólica dos méis analisados, fator que indica possível seletividade das espécies em relação à origem botânica dos méis.

De modo geral, os méis analisados apresentaram uma significativa atividade antioxidante, principalmente os méis mais escuros que possuíram também teores mais elevados de polifenóis totais.

\section{MATERIAL SUPLEMENTAR}

Os cromatogramas de ácidos fenólicos e flavonoides encontrados nos méis das espécies do estudo estão disponíveis gratuitamente em http://quimicanova.sbq.org.br, na forma de arquivo PDF, com acesso livre.

\section{AGRADECIMENTOS}

À Fundação de Apoio à Pesquisa do Estado do Pará (FAPESPA) pela bolsa de mestrado de P. S. Oliveira e apoio financeiro (Projeto FAPESPA UNIVERSAL 101).

\section{REFERÊNCIAS}

1. Engelsdorpa, D. V.; Meixnerb, M. D.; J. Inv. Pathol. 2010, 103, 80.

2. Venturieri, G. C.; Raiol, V. F.O.; Pereira, C. A. B.; Rev. Biota Neotrop. 2003, 3, 27.

3. Rasmussen, C.; Cameron, S. A.; Systematic Entomology 2007, 32, 26.

4. Cortopassi-Laurino, M.; Apidologie 2006, $37,1$.

5. Roubik, D. W.; Ecology and Natural History of Tropical Bees, Cambridge University Press: New York, 1989.

6. Paar, A. J. E.; Bolwell, G. P.; J. Sci. Agric. 2000, 80, 985; Rhodes, M. J. C.; Plant. Mol. Biol. 1994, 24, 1; Slattery, M. L.; Benson, J.; Curtin, K.; Ma, K. N.; Schaeffer, D.; Potter, J. D.; J. Am. Clin. Nutr. 2000, 71, 575; Steinmetz, K. A.; Potter, J. D.; J. Am. Diet. Assoc. 1996, 96, 1027.

7. Cortopassi-Laurino, M.; Resumos do $6^{\circ}$ Seminário Nordestino de Pecuária, Fortaleza, Brasil, 2002.

8. Kerr, W. E.; Carvalho, G. A.; Nascimento, V. A.; A abelha uruçu: biologia, manejo e conservação, Acanjaú: Belo Horizonte, 1996.

9. Nogueira-Neto, P.; Vida e Criação de Abelhas Indígenas Sem Ferrão, Nogueirapis: São Paulo, 1997.

10. http://sistemasdeproducao.cnptia.embrapa.br/SPMel, acessada em Maio 2009.

11. Campos, G.; Modesta, R. C. D.; Rev. IAL 2000, 59, 7; Azeredo, M. A. A.; Azeredo, L. da C.; Damasceno, J. G.; Ciênc. e Tecnol. Aliment. 1999, 19, 3; Pamplona, B. C.; Dissertação de Mestrado, Universidade de São Paulo, Brasil, 1989; Al-Mamary, M.; Al-Meeri, A.; Al-Habori, A.; Nutr. Res. 2002, 22, 1041; Andrade, P.; Ferreres, F.; Amaral, M. T.; J. Liq. Chromatogr. Relat. Technol. 1997, 14, 2281; D’Arcy, R. B.; Rural Industries Research and Development Corporation, Project No. UQ-102a , 2005, 84; Estevinho, L; Pereira, A. P.; Moreira, L.; Dias, L. G.; Pereira, E.; Food Chem. Toxicol. 2008, 46, 3774; Ferreira, C. F. R. I.; Aires, E.; Barreira, C. M. J.; Meda, A.; Lamien, E. C.; Romito, M.; Millogo, J.; Nacoulma, G. O.; Food Chem. 2005, 91, 571.

12. Estevinho, M. L.; Food Chem. 2009, 114, 1438.

13. Lianda, R. L. P.; Castro, R. N.; Echevarria, A.; Resumos da $32^{a}$ Reunião Anual da Sociedade Brasileira de Química, Fortaleza, Brasil, 2009.

14. Silva, T. M. S.; Camara, C. A.; Lins, A. C. S.; Barbosa-Filho, J. M.; Silva, E. M. S.; Freitas, B. M.; Santos, F. A. R.; J. Food Compos. Anal. 2005, 19, 507.

15. Bravo, L.; Abia, R. Y.; Saura-Calixto, F.; J. Agric. Food Chem. 1994, 42, 1481.

16. Alves, R. M. de O.; Souza, B. de A.; Carvalho, C. A. L. de; Justina, G. D.; Criação de abelhas, SEAGRI: Cruz das Almas, 2005; Yao, L.; Jiang, Y.; Singanusong, R.; Datta, N.; Raymont, K.; Food Chem. 2004, 86, 169; Kwakman, P. H. S.; Velde, A. A.; Boer, L.; Speijer, D.; VandenbrouckeGrauls, C. M. J. E.; Zaat, S. A. J.; The FASEB J. 2010, 24.

17. Yao, L.; Datta, N.; Tomás-Barberán, F. A.; Ferreres, F.; Martos, I.; Singanusong, R.; Food Chem. 2003, 81, 159.

18. Michalkiewicz, A.; Biesaga, M.; Pyrzynska, K.; J. Chromatogr., A 2008, $1187,18$.

19. Kowalski, B. R.; Bender, C. F.; Anal. Chem. 1972, 44, 1045.

20. Venturieri, G. C.; Criação de abelhas indígenas sem ferrão, Embrapa Amazônia Oriental: Belém, 2005, p. 31-32.

21. Venturieri, G. C.; Oliveira, P. S.; Vasconcelos, M. A. M.; Mattietto, R. A.; Caracterização, colheita, conservação e embalagem de méis de abelhas indígenas sem ferrão, Embrapa Amazônia Oriental: Belém, 2007.

22. Carvalho, A. S. C.; Santos, A. S.; Pereira, S. F. P.; Alves, C. N.; J. Braz. Chem. Soc. 2009, 20, 1163.

23. Sharaf, M. A.; Ilman, D. L.; Kowalski, B. R.; Chemometrics, $1^{\text {st }}$ ed., Wiley: New York, 1986.

24. Minitab Release 14; State College, Pennsylvania, Inc, 2004.

25. Vidal, R.; Fregosi, E. V.; Mel: Características, análises físico-químicas, adulterações e transformações, ITC Roberto Rios: Barretos, 1984, p. 95. 
ÁCIDOS FENÓLICOS, FLAVONOIDES E ATIVIDADE ANTIOXIDANTE EM MÉIS DE Melipona fasciculata, M. flavolineata (APIDAE, MELIPONINI) E Apis Mellifera (APIDAE, APINI) DA AMAZÔNIA

Patricia Sertão Oliveira, Regina Celi Sarkis Müller*, Kelly das Graças Fernandes Dantas e Claudio Nahum Alves Faculdade de Química, Instituto de Ciências Exatas e Naturais, Universidade Federal do Pará, Rua Augusto Corrêa, 01, 66075-110 Belém - PA, Brasil

Marcus Arthur Marçal de Vasconcelos e Giorgio Cristino Venturieri

Embrapa Amazônia Oriental, Trav. Enéias Pinheiro, s/n, 66017-970 Belém - PA, Brasil
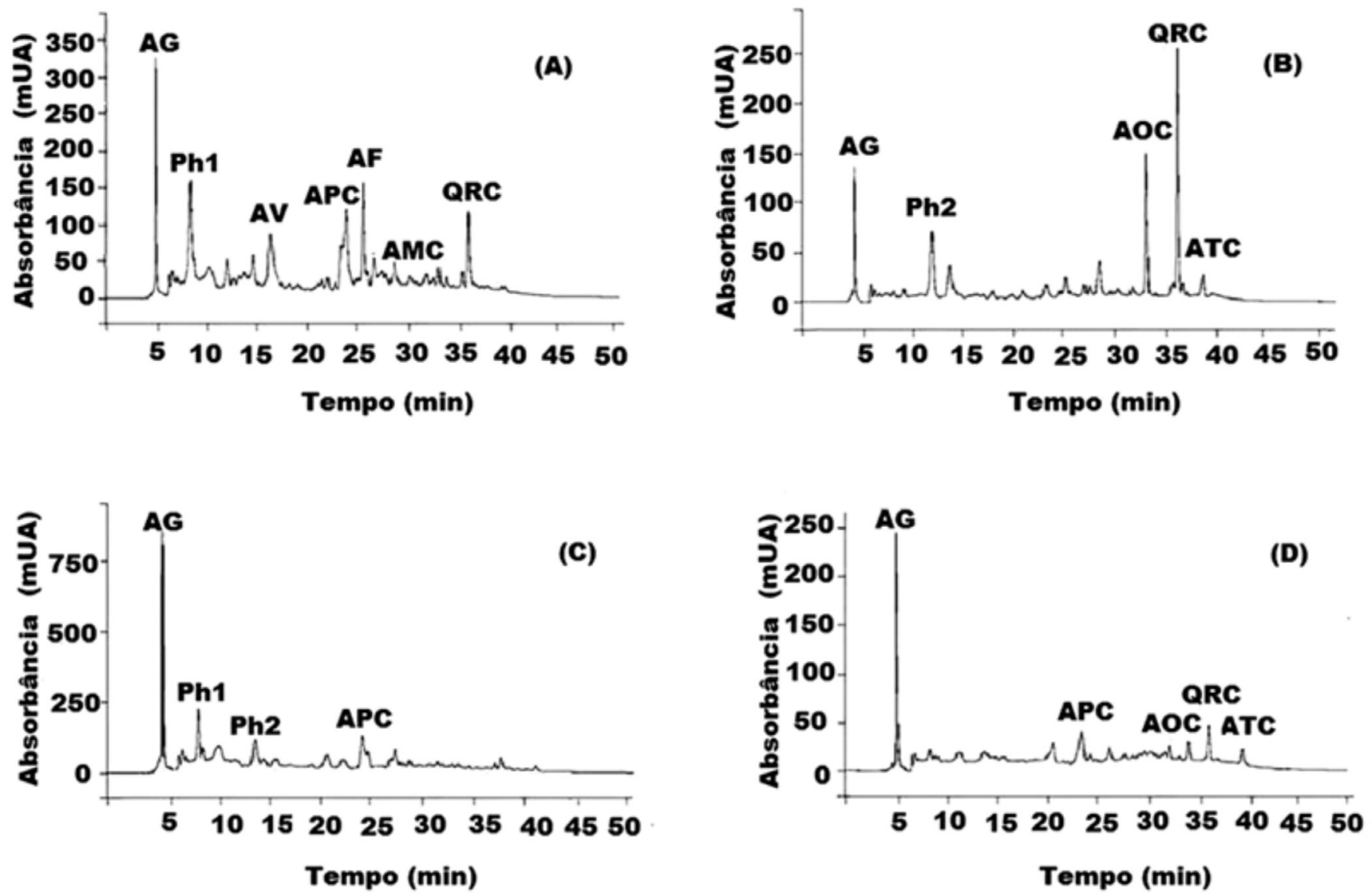

Figura 1S. Cromatograma de ácidos fenólicos e flavonoides através de RP-HPLC a $290 \mathrm{~nm}$. A. mellifera. Amostras de Tracuateua (A): AG, "Ph, AV, APC, $M R C, A F, A M C, Q R C$, *Ph-ácido fenólico desconhecido; amostras de São João de Pirabas (B): $A G, P h_{1}, P h_{2}, A P C, A F, A O C, Q R C, A T C$; amostras de São Miguel do Guamá (C): $A G, P h_{1}, P h_{2}, A P C, M R C$; amostras de Vigia (D): AG, APC, AOC, QRC, ATC 

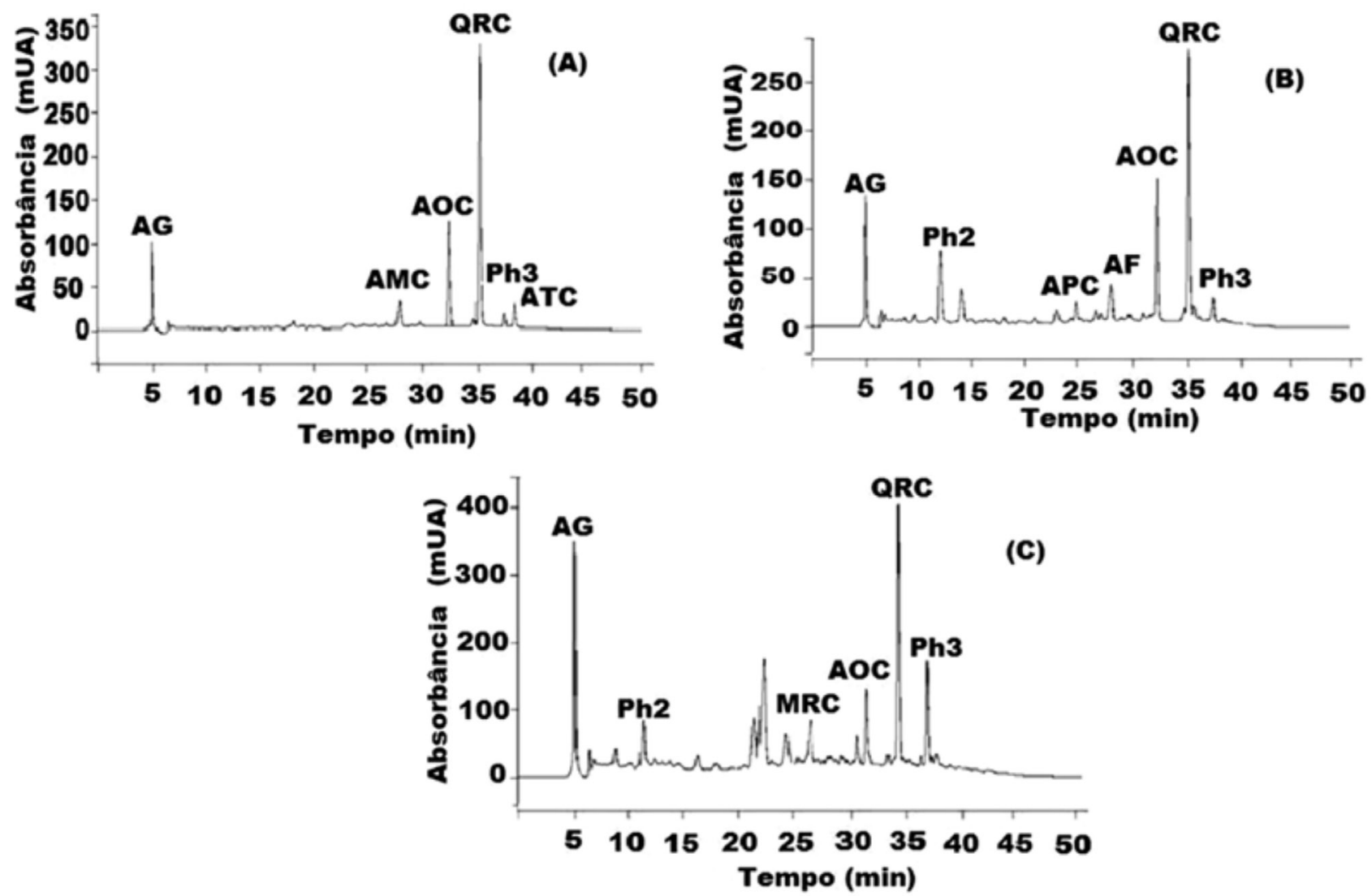

Figura 2S. Cromatograma de ácidos fenólicos e flavonoides através de RP-HPLC a 290 nm. M. flavolineata. Amostras de Tracuateua (A): AG ácido gálico, AMC, AOC, $Q R C, P h_{3}$, ATC; amostras de São João de Pirabas (B): $A G, P h_{l}, P h_{2}, A P C, A F, A O C, Q R C, A T C$; amostras de Vigia $(C): A G, P h_{l}, P h_{2}, A P C, A O C, Q R C, P h_{3}$
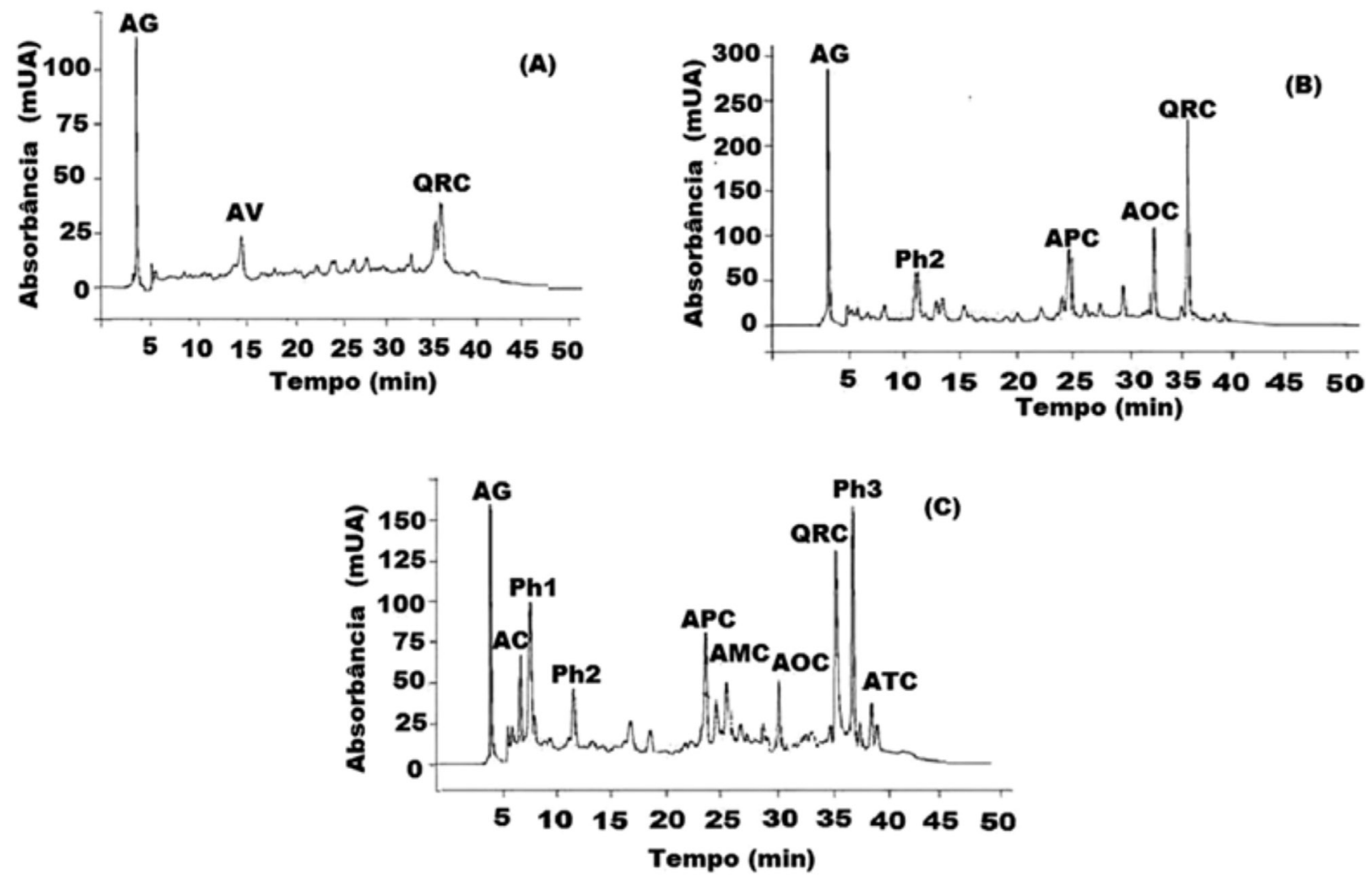

Figura 3S. Cromatograma de ácidos fenólicos e flavonoides através de RP-HPLC a $290 \mathrm{~nm}$. M. fasciculata. Amostras de Tracuateua (A): AG, Ph ${ }_{1}, P h_{2}$ APC, $A O C, Q R C, \mathrm{Ph}_{3}$; amostras de São João de Pirabas (B): $A G, P h_{l}, A P C, A F, A O C, Q R C$; amostras de São Miguel do Guamá (C): $A G$ e Ph 